\title{
The self-dual classical double copy, and the Eguchi-Hanson instanton
}

\section{David S. Berman, ${ }^{a}$ Erick Chacón, ${ }^{b}$ Andrés Luna ${ }^{c}$ and Chris D. White ${ }^{a}$}

${ }^{a}$ Centre for Research in String Theory, School of Physics and Astronomy, Queen Mary University of London, 327 Mile End Road, London E1 4NS, U.K.

${ }^{b}$ Departamento de Fúsica, Centro de Investigación y de Estudios Avanzados del Instituto Politécnico Nacional, P.O. Box 14-740, CP. 07000, México D.F., México

${ }^{c}$ Mani L. Bhaumik Institute for Theoretical Physics, Department of Physics and Astronomy, University of California, Los Angeles, California 90095, U.S.A.

E-mail: d.s.berman@qmul.ac.uk, echacon@fis.cinvestav.mx, andreslunagodoy@hotmail.com, christopher.white@qmul.ac.uk

ABSTRACT: The double copy is a map from non-abelian gauge theories to gravity, that has been demonstrated both for scattering amplitudes and exact classical solutions. In this study, we reconsider the double copy for exact solutions that are self-dual in either the gauge or gravity theory. In this case, one may formulate a general double copy in terms of a certain differential operator, which generates the gauge and gravity solutions from a harmonic function residing in a biadjoint scalar theory. As an illustration, we examine the single copy of the well-known Eguchi-Hanson instanton in gravity. The gauge field thus obtained represents an abelian-like object whose field is dipole-like at large distances, and which has no magnetic or electric charge.

KEYwords: Scattering Amplitudes, Solitons Monopoles and Instantons

ARXIV EPRINT: 1809.04063 


\section{Contents}

1 Introduction 1

2 Self-dual Kerr-Schild solutions 3

$\begin{array}{llr}3 & \text { The Eguchi-Hanson solution } & 6\end{array}$

4 Conclusion $\quad 12$

\section{Introduction}

Classical and quantum aspects of various field theories continue to be intensely studied, due to a plethora of applications in astro-, high energy and condensed matter physics. Particularly interesting in recent times have been newly discovered correspondences between different theories, whose domains of application in nature are widely separated. In this paper, we focus on the double copy [1-3], a correspondence between gauge theories and gravity. ${ }^{1}$ In its original form, the double copy relates perturbative scattering amplitudes in the two types of theory, subject to a certain mirroring of colour and kinematic information, $B C J$ duality [1], being made manifest in the gauge theory. The relationship is proven to hold at tree level [3-11], where it has a string theoretic explanation [12]. Although a full loop level proof has not been found, there is highly non-trivial evidence that the double copy works at higher orders in perturbation theory in a wide variety of theories (with and without supersymmetry) [2,13-35], including all-order evidence in some cases [36-43]. Another relationship, the zeroth copy, relates amplitudes in gauge theory to those in a biadjoint scalar theory, i.e. containing a scalar field interacting via two different types of colour charge.

The highly non-trivial nature of the above results has prompted many to ponder whether the double and zeroth copies are an accident of scattering amplitudes in perturbation theory, or instead indicative of a deeper - and hitherto undiscovered - connection between biadjoint scalar, gauge and gravity theories. Indeed, the double and zeroth copies can be extended to a class of exact classical solutions. The best understood family is that of time-independent Kerr-Schild metrics in gravity [44], where the graviton field has a highly specific form involving an outer product of a single null vector. Extensions to this class have been considered in [45, 46], where the single Kerr-Schild and / or time independence properties can be at least partially relaxed (see also ref. [47] for a discussion of whether the source terms in the gauge and gravity theory are related, in addition to the fields). Perturbative classical solutions have also been examined: refs. [48-56] set up a broad programme

\footnotetext{
${ }^{1}$ Throughout, we use the term gauge theory to refer to abelian or non-abelian gauge theories, as distinct from gravity, unless otherwise stated.
} 
for identifying linearised fields in a wide variety of exotic theories, and in a gauge-invariant manner. Furthermore, classical scattering processes can be double copied order-by-order in perturbation theory - see ref. [57] and the related refs. [58-67] — which promises to vastly streamline the calculation of astrophysically relevant observables in classical General Relativity, such as those related to gravitational waves. In a wider context, the double and zeroth copies have a natural embedding in the so-called CHY equations [68, 69], which themselves are obtainable from ambitwistor string theory [70]. These and other considerations have recently led to extensions of the copies to curved spacetimes [71-73], and to double field theory [74]. Recently, the double copy has been used to motivate the algebraic classification of higher-dimensional gravity solutions [75].

Despite these ongoing efforts, a full understanding of the scope and generality of the double copy remains elusive. This underlines the importance of having different ways of thinking about the copy where it applies, and of looking at different sectors of gauge and gravity theories for which the copy is particularly clear. One such sector is that of solutions to the equations of motion where the field is self-dual, which has the effect of projecting out one of the physical polarisation states of the gluon or graviton. It was shown some time ago that the amplitude double copy has a particularly natural form in the self-dual sector $[10,11,76]$, with the added bonus that the BCJ duality property required for gauge theory amplitudes to satisfy the double copy can be fully interpreted. This already suggests that a reexamination of the self-dual sector could be fruitful for gaining new insights. Furthermore, the first paper to explore the double copy of exact classical solutions [44] pointed out that the properties of self-dual amplitudes can be recast in a KerrSchild language, but where the null vectors are interpreted as differential operators. The motivation for exploring the self-dual sector in ref. [44] was to demonstrate that the KerrSchild copy for classical solutions was merely a different manifestation of the previously described double copy for amplitudes of refs. [1-3]. However, implications of the self-dual Kerr-Schild double copy for classical solutions were not followed up, and it is the aim of this paper to explore this in more detail.

Remarkably, a related story for exact classical solutions goes all the way back to ref. [77], which realised that null electromagnetic fields could be associated with self-dual Kerr-Schild gravity metrics. We will review this argument in what follows, interpreting the results from a modern viewpoint. More specifically, we will see that given a harmonic scalar function, we may apply a particular vector differential operator to generate a self-dual solution to the Maxwell equations. A second application of the same operator generates a Kerr-Schild graviton, and thus one generates a ladder of fields residing in biadjoint scalar, gauge and gravity theories, all of whose equations linearise. That this is consistent with the BCJ double copy for amplitudes follows from the results of ref. [44].

As an example of the procedure, we examine a particular harmonic function (first studied in ref. [77]) that gives rise to the Eguchi-Hanson solution [78-80], which enjoys a well-known interpretation as a gravitational instanton. We use the self-dual Kerr-Schild approach to study the single copy of this solution. The single copy is a solution of the (abelian) Maxwell equations, whose electric and magnetic fields are dipole-like at large distances. Unlike previously considered solutions, however, there is no magnetic charge, 
nor (by self-duality) any electric charge anywhere in space. Furthermore, the fact that the single copy is abelian-like rather than non-abelian is itself interesting, given that one might have expected the single copy of the Eguchi-Hanson instanton to be a non-abelian instanton e.g. the BPST instanton of ref. [81]. Parallels between these instanton solutions were noted even at the inception of the Eguchi-Hanson solution [78] (see e.g. ref. [82] for a more modern review, and refs. [83, 84] for studies in the context of noncommutative theories). Our results will thus have interesting implications for attempts to study non-perturbative aspects of the double copy, including those that attempt to identify symmetries and / or topological invariants in gauge and gravity theories.

The structure of our paper is as follows. We briefly review the Kerr-Schild double copy in section 2, including aspects relating to the self-dual sector. In section 3 , we examine in detail the single and zeroth copies of the Eguchi-Hanson solution, including a discussion of topological properties. We discuss our results and conclude in section 4 .

\section{Self-dual Kerr-Schild solutions}

In this section, we briefly review the properties of the Kerr-Schild double copy, including its formulation tailored to self-dual solutions. More details can be found in ref. [44]. We start by considering a solution $g_{\mu \nu}$ to Einstein gravity. Expanding around flat space, we may define a graviton field $h_{\mu \nu}$ via

$$
g_{\mu \nu}=\eta_{\mu \nu}+\kappa h_{\mu \nu}
$$

where $\eta_{\mu \nu}$ is the Minkowski metric, and $\kappa^{2}=16 \pi G_{N}$, with $G_{N}$ the Newton constant. The metric $g_{\mu \nu}$ is of Kerr-Schild form if the graviton field can be written as

$$
h_{\mu \nu}=\Phi k_{\mu} k_{\nu}
$$

where $\Phi$ is a scalar field, and $k_{\mu}$ is null and geodesic:

$$
k^{2}=0, \quad k \cdot \partial k^{\mu}=0 .
$$

Upon substituting the ansatz of eqs. (2.1)-(2.3) into the Einstein equations, one finds that the Ricci tensor $R^{\mu}{ }_{\nu}$ truncates at linear order in $\kappa$, so that the graviton obtained from $\Phi$ and $k_{\mu}$ constitutes an exact gravitational solution. Reference [44] proved that, given any time-independent Kerr-Schild graviton, the vector field

$$
A_{\mu}^{a}=c^{a} \Phi k_{\mu}
$$

(where $c^{a}$ is an arbitrary colour vector) is a solution of the abelian Yang-Mills (Maxwell) equations

$$
\partial^{\mu} F_{\mu \nu}^{a}=0 .
$$

Thus, any such gravity solution has a well-defined gauge theory counterpart, which can be interpreted as the "single copy" of the former. Furthermore, upon repeating the procedure 
of replacing one of the null vectors $k^{\mu}$ with an arbitrary colour vector, one obtains the single scalar field

$$
\Phi^{a a^{\prime}}=c^{a} \tilde{c}^{a^{\prime}} \Phi,
$$

that is found to satisfy the linearised biadjoint scalar field equation

$$
\partial^{2} \Phi^{a a^{\prime}}=0 .
$$

Thus, the field $\Phi^{a a^{\prime}}$ is to be regarded as the "zeroth copy" associated with the gravity solution $h_{\mu \nu}$ and gauge field $A_{\mu}$, and a number of non-trivial examples of this correspondence were given in ref. [44], including the Schwarzschild and Kerr black holes. Extensions to include multiple Kerr-Schild-like terms were considered in ref. [45], for which the TaubNUT solution $[85,86]$ was found to be a special case. Time dependence was also added in ref. [46], which considered an arbitrarily accelerating point charge (mass) in gauge theory (gravity). Known amplitudes for photon and graviton Bremstrahlung were recovered as part of this picture, making clear that the Kerr-Schild double copy for classical solutions is a manifestation of the same double copy that relates scattering amplitudes in biadjoint scalar, gauge and gravity theories.

In this paper, we focus on solutions that are self-dual, namely those satisfying (in Euclidean space)

$$
F_{\mu \nu}=\tilde{F}_{\mu \nu} \equiv \frac{\sqrt{\operatorname{det} g}}{2} \epsilon_{\mu \nu \rho \sigma} F^{\rho \sigma}
$$

and

$$
R_{\mu \nu \lambda \sigma}=\tilde{R}_{\mu \nu \lambda \sigma} \equiv \frac{\sqrt{\operatorname{det} g}}{2} \epsilon_{\mu \nu \rho \sigma} R_{\lambda \sigma}^{\rho \sigma}
$$

in gauge theory and gravity respectively, where $F_{\mu \nu}\left(R_{\mu \nu \lambda \sigma}\right)$ is the field strength tensor (Riemann curvature), and $\epsilon_{\mu \nu \rho \sigma}$ the Levi-Cevita symbol. Physically, such solutions correspond to keeping only one of the two physical polarisation states of the photon or graviton, and for such solutions one may think about the Kerr-Schild double copy in a different way. Namely, one may formulate the Kerr-Schild vector $k_{\mu}$ as a differential operator $\hat{k}_{\mu}$, which amounts to considering it as multiplicative in momentum rather than position space. The symmetry of the resulting graviton

$$
h_{\mu \nu}=\hat{k}_{\mu} \hat{k}_{\nu} \Phi
$$

then implies that $\left[\hat{k}_{\mu}, \hat{k}_{\nu}\right]=0$ i.e. $\hat{k}_{\mu}$ commutes with itself. Furthermore, we may implement the null and geodesic Kerr-Schild conditions as

$$
\eta_{\mu \nu} \hat{k}^{\mu} \hat{k}^{\nu}=0, \quad \hat{k} \cdot \partial=0 .
$$

A suitable operator can be defined by adopting the lightcone coordinates

$$
u=\frac{t-i z}{\sqrt{2}}, \quad v=\frac{t+i z}{\sqrt{2}}, \quad X=\frac{i x-y}{\sqrt{2}}, \quad Y=\frac{i x+y}{\sqrt{2}},
$$

where, following ref. [77], we define $(u, v, X, Y)$ in terms of Euclidean Cartesian coordinates $(t, x, y, z)$ in $\mathbb{R}^{4}$. The operator $\hat{k}_{\mu}$ may now be chosen as

$$
\hat{k}_{u}=\partial_{X}, \quad k_{v}=0, \quad k_{X}=0, \quad k_{Y}=\partial_{v},
$$


and we may then show that the Einstein equations for the graviton of eq. (2.10) reduce to

$$
\partial^{2} \Phi+\kappa\left\{\partial_{X} \Phi, \partial_{u} \Phi\right\}=0
$$

where we introduced the Poisson bracket of two functions $f$ and $g$ :

$$
\{f, g\}=\left(\partial_{X} f\right)\left(\partial_{u} g\right)-\left(\partial_{u} f\right)\left(\partial_{X} g\right) .
$$

Equation (2.14) is the Plebanski equation describing self-dual gravity, and a similar equation can be obtained for Yang-Mills theory by considering the Kerr-Schild single copy field

$$
A_{\mu}^{a}=\hat{k}_{\mu} \Phi^{a}
$$

for an adjoint-valued scalar field $\Phi=\Phi^{a} \mathbf{T}^{a}$, where $\mathbf{T}^{a}$ is a generator of the gauge group. With this ansatz, the Yang-Mills equations turn out to imply

$$
\partial^{2} \Phi+2 i g\left[\partial_{v} \Phi, \partial_{X} \Phi\right]=0
$$

a known equation ${ }^{2}$ for self-dual Yang-Mills theory first derived in ref. [87], and studied within a double copy context in ref. [10], where it was used to argue that amplitudes in the self-dual sector are manifest double copies of each other. Furthermore, a kinematic algebra underlying the numerators of self-dual amplitudes was obtained, that provided an explicit realisation of the BCJ duality between colour and kinematic degrees of freedom. The identification of eq. (2.16) makes clear that the Kerr-Schild single copy for self-dual solutions is indeed the same as the double copy as originally formulated for amplitudes. However, it can also be noted that eq. (2.16) is equally valid for exact classical solutions. In this context, this approach is actually a modern reinterpretation of the much earlier ref. [77], which also recognised that self-dual Kerr-Schild gravitons could be associated with null Maxwell fields.

We may adapt the argument of ref. [77] to the above discussion as follows. First, we rewrite the Plebanski equation of eq. (2.14) as

$$
\Phi_{, u v}-\Phi_{, X Y}=-\Phi_{, v v} \Phi_{, X X}+\left(\Phi_{, v X}\right)^{2},
$$

where the comma denotes the partial derivative. The metric of eq. (2.10) can be written

$$
d s^{2}=2 d u d v-2 d X d Y-2 \kappa\left(\Phi_{, X X} d u^{2}+2 \Phi_{, v X} d u d Y+\Phi_{, v v} d Y^{2}\right),
$$

where we have used the form of the Minkowski metric

$$
\eta_{\mu \nu}=2 d u d v-2 d X d Y
$$

in the lightcone coordinates of eq. (2.12). However, if the metric of eq. (2.20) is to be in Kerr-Schild form, then the $\mathcal{O}(\kappa)$ term must be a perfect square, which in turn implies

$$
\Phi_{, v v} \Phi_{, X X}-\left(\Phi_{, v X}\right)^{2}=0 .
$$

\footnotetext{
${ }^{2}$ The form of eq. (2.17) differs slightly from that given in refs. [76, 87], due to different conventions for the lightcone coordinates and Kerr-Schild operator.
} 
Coupled with the requirement of self-duality, eq. (2.18), this implies that the function $\Phi$ must be harmonic. But this is none other than the requirement that the field $\Phi$ satisfy the linearised biadjoint scalar equation of eq. (2.7). Thus, we can summarise the results of ref. [77] from a double copy point of view as follows: given a harmonic function $\Phi$, we may consider the fields

$$
\Phi, \quad A_{\mu}=\hat{k}_{\mu} \Phi, \quad h_{\mu \nu}=\hat{k}_{\mu} \hat{k}_{\nu} \Phi,
$$

living in a biadjoint scalar, gauge and gravity theory respectively. ${ }^{3}$ The fields $A_{\mu}$ and $\Phi$ constitute the classical single and zeroth copies of the graviton $h_{\mu \nu}$ respectively. Having set up the general framework for self-dual classical solutions, we now consider a particular example in detail.

\section{The Eguchi-Hanson solution}

In the previous section, we described a procedure for generating gauge theory and graviton fields obeying the double copy, by acting upon a harmonic function in lightcone coordinates with the differential operator of eq. (2.13). In this section, we study a particular example in detail, namely the Eguchi-Hanson solution of Euclidean gravity, originally derived in refs. [78], and interpreted in more detail in refs. [79, 80, 88]. A conventional way to present this solution is to choose a radial coordinate $r$ in $\mathbb{R}^{4}$, and three Euler angles

$$
0 \leq \theta \leq \pi, \quad 0 \leq \phi \leq 2 \pi, \quad 0 \leq \psi \leq 4 \pi,
$$

such that the line element may be written

$$
d s_{\mathrm{EH}}^{2}=\left[1-(a / r)^{4}\right]^{-1} d r^{2}+r^{2}\left\{\sigma_{x}^{2}+\sigma_{y}^{2}+\left[1-(a / r)^{4}\right] \sigma_{z}^{2}\right\},
$$

where

$$
\begin{aligned}
\sigma_{x} & =\frac{1}{2}(\sin \psi d \theta-\sin \theta \cos \psi d \phi), \\
\sigma_{y} & =\frac{1}{2}(-\cos \psi d \theta-\sin \theta \sin \psi d \phi), \\
\sigma_{z} & =\frac{1}{2}(d \psi+\cos \theta d \phi) .
\end{aligned}
$$

With this choice of coordinates, the topology of the spacetime is that of $\mathbb{R}_{+} \times S^{3}$, although it has a singularity at $r=a$. We can characterise the topology in four spacetime dimensions using two invariants, namely the Hirzebruch signature $\tau$ and the Euler characteristic $\chi$ (see e.g. [89] for a review). These receive contributions from the bulk of the spacetime, and the boundary, and are both zero for $\mathbb{R}_{+} \times S^{3}$ [78] (n.b. to get this result, it is crucial that one includes the boundary contribution from the singularity at $r=a$ ).

However, one can also construct a topologically non-trivial spacetime from the above metric, by removing the singularity at $r=a$ [79]. Changing variables to

$$
u=r^{2}\left[1-(a / r)^{4}\right]
$$

\footnotetext{
${ }^{3}$ Strictly speaking, we must dress the biadjoint and gauge fields with arbitrary colour vectors as in eqs. (2.4), (2.6). However, given that the equations of motion linearise in each theory for the solutions considered, we can ignore this unless otherwise stated.
} 
and expanding near $u=0(r=a)$, one finds

$$
d s_{\mathrm{EH}}^{2} \simeq \frac{1}{4} d u^{2}+\frac{1}{4} u^{2}(d \psi+\cos \theta d \phi)^{2}+\frac{a^{2}}{4}\left(d \theta^{2}+\sin ^{2} \theta d \phi^{2}\right),
$$

such that the local topology near $r=a$ is that of $\mathbb{R}^{2} \times S^{2}$, where the latter factor is associated with the coordinates $(\theta, \phi)$. At fixed values of these coordinates one has the pure $\mathbb{R}^{2}$ metric

$$
\left.d s_{\mathrm{EH}}^{2}\right|_{\theta=\phi=\text { const. }} \simeq \frac{1}{4}\left(d u^{2}+u^{2} d \psi^{2}\right),
$$

whose singularity at $u=0$ is removable provided one restricts the Euler angle $\psi$ to lie in the range

$$
0 \leq \psi \leq 2 \pi
$$

rather than the full range of eq. (3.1). Put another way, this has the effect of identifying each spacetime point $x^{\mu}$ with $-x^{\mu}$, so that the boundary of the spacetime becomes the projective space $P_{3}(\mathbb{R})=S^{3} / Z_{2}$ rather than $S^{3}$. Recalculation of the topological invariants mentioned above then yields $\chi=2, \tau=1$. Removable singularities of this type were christened bolts in ref. [90], as distinct from (anti)-nut singularities (such as those in the Taub-NUT metric studied in a double copy context in ref. [45]). The invariants $\chi$ and $\tau$ then have an interpretation in terms of a superposition of the number of nuts and bolts [90]. The spacetime obtained by removing the bolt singularity in the present case has zero action, is everywhere regular, and is also self-dual. It has thus been widely studied as an example of a gravitational instanton.

Let us now turn, as promised, to the single copy of the Eguchi-Hanson solution, for which we first need to know the zeroth copy field $\Phi$. Following section 2 , this is a harmonic function residing in a biadjoint scalar field theory, that one must act upon with the KerrSchild operator of eq. (2.13) in order to produce a gauge field and, subsequently, the Eguchi-Hanson graviton. Furthermore, this function must be given in terms of the lightcone coordinates of eq. (2.12). The relevant function has been given in ref. [77] as

$$
\Phi=\frac{\lambda X^{2}}{2 u^{2}(u v-X Y)},
$$

from which one obtains the gauge field

$$
A_{\mu}=\hat{k}_{\mu} \Phi=\lambda \frac{X^{2}}{2 u^{2}(u v-X Y)^{2}}\left(\frac{2 u v-X Y}{X}, 0,0,-u\right)
$$

and graviton

$$
h_{\mu \nu}=\lambda \frac{(v d u-X d Y)^{2}}{(u v-X Y)^{3}} .
$$

Here we have included a common parameter $\lambda$, so that vacuum solutions in each theory correspond to $\lambda \rightarrow 0$. We do not include explicit factors of the coupling in the gauge or biadjoint theories, choosing instead to absorb these in the arbitrary colour vectors that would dress the above solutions. 
Let us first check that eq. (3.10) indeed corresponds to the Eguchi-Hanson metric of eq. (3.2), which we may write more clearly by substituting eq. (3.3) into eq. (3.2):

$$
d s_{\mathrm{EH}}^{2}=\frac{1}{4} r^{2}\left[1-(a / r)^{4}\right][d \psi+\cos \theta d \phi]^{2}+\left[1-(a / r)^{4}\right]^{-1} d r^{2}+\frac{1}{4} r^{2}\left[d \theta^{2}+\sin ^{2} \theta d \phi^{2}\right] .
$$

This equivalence has been shown using twistor methods in ref. [91], but we can instead use a more straightforward approach. Adding the background flat line element (in lightcone coordinates) to eq. (3.10), one obtains

$$
d s^{2}=2 d u d v-2 d X d Y+\lambda \frac{(v d u-X d Y)^{2}}{(u v-X Y)^{3}} .
$$

The lightcone coordinates may be transformed to (Euclidean) Cartesian coordinates according to eq. (2.12), after which one may make the further transformation

$$
\begin{aligned}
& x=r \cos \left(\frac{\theta}{2}\right) \cos \left(\frac{\phi+\psi}{2}\right), \\
& y=r \cos \left(\frac{\theta}{2}\right) \sin \left(\frac{\phi+\psi}{2}\right), \\
& z=r \sin \left(\frac{\theta}{2}\right) \cos \left(\frac{\phi-\psi}{2}\right), \\
& t=r \sin \left(\frac{\theta}{2}\right) \sin \left(\frac{\phi-\psi}{2}\right) .
\end{aligned}
$$

Here $r$ is the radial distance in $\mathbb{R}^{4}$, and from the two sets of transformations in eqs. (2.12), (3.13) we can establish the relation

$$
r^{2}=x^{2}+y^{2}+z^{2}+t^{2}=2(u v-X Y) .
$$

Next, we can connect the parameter $\lambda$ from eq. (3.10) with the parameter $a$ appearing in the metric of eq. (3.2), by considering the Kretschmann scalar in both the lightcone and polar coordinate systems:

$$
R^{\alpha \beta \mu \nu} R_{\alpha \beta \mu \nu}=\frac{384 a^{8}}{r^{12}}=\frac{24 \lambda^{2}}{(u v-X Y)^{6}} .
$$

Equation (3.14) then immediately implies

$$
\lambda=\frac{a^{4}}{2}
$$

such that transforming eq. (3.12) into the polar coordinate system of eq. (3.13) yields

$$
d s^{2}=d r^{2}+\frac{1}{4} r^{2}\left[d \psi^{2}+d \phi^{2}+d \theta^{2}+2 \cos \theta d \psi d \phi\right]+\frac{4 a^{4}}{r^{6}}\left(\frac{r d r}{2}-\frac{i r^{2}}{4}(d \psi+\cos \theta d \phi)\right)^{2}
$$


Finally, one may perform the additional transformation

$$
\psi \rightarrow \psi+\frac{i}{2} \log \left(\frac{r^{4}-a^{4}}{r^{4}}\right)
$$

to put eq. (3.17) into the form of eq. (3.2). Note that this last transformation is singular, and creates the explicit coordinate singularity at $r=a$ (the "bolt") appearing in eq. (3.2). This final coordinate transformation diagonalises the metric, after which all angles have the ranges specified in eq. (3.1).

Armed with the above transformations, we can also transform the zeroth copy field $\Phi$ and single copy gauge field $A_{\mu}$ of eqs. (3.8), (3.9) into the polar coordinate system, and we find

$$
\Phi=\frac{r^{2}}{r^{4}-a^{4}} \cot ^{2}(\theta / 2) e^{2 i \psi},
$$

and

$$
A_{\mu}=\frac{e^{i \psi}}{\left(r^{4}-a^{4}\right)^{1 / 2}}\left(\frac{2 r^{3} \cot (\theta / 2)}{a^{4}-r^{4}},-\frac{1}{2} \cot ^{2}(\theta / 2), i \cot (\theta / 2),-\frac{i}{2} \sin (\theta)\right) .
$$

We may also calculate the gauge-invariant field strength tensor,

$$
F_{\mu \nu}=\partial_{[\mu} A_{\nu]}=\frac{e^{i \psi}}{\sqrt{r^{4}-a^{4}}}\left(\begin{array}{cccc}
0 & -\frac{r^{3}}{r^{4}-a^{4}} & 0 & \frac{i r^{3} \sin \theta}{r^{4}-a^{4}} \\
\frac{r^{3}}{r^{4}-a^{4}} & 0 & -\frac{i}{2} & -\frac{i \cos \theta}{2} \\
0 & \frac{i}{2} & 0 & \frac{\sin \theta}{2} \\
-\frac{i r^{3} \sin \theta}{r^{4}-a^{4}} & \frac{i \cos \theta}{2} & -\frac{\sin \theta}{2} & 0
\end{array}\right) .
$$

As a cross-check, we have explicitly verified (in the final coordinate system) that eq. (3.21) satisfies the Maxwell equations

$$
D_{\mu} F^{\mu \nu}=0
$$

where $D_{\mu}$ is the covariant derivative in the curvilinear coordinate system obtained by transforming the flat space metric from the lightcone coordinate system via eqs. (2.12), (3.13), (3.18), as well as the appropriate self-duality condition

$$
F_{\mu \nu}=-\frac{\sqrt{g}}{2} \epsilon_{\mu \nu \alpha \beta} F^{\alpha \beta},
$$

where $g$ is the determinant of the metric. One may also verify that

$$
F_{\mu \nu} F^{\mu \nu}=F_{\mu \nu} \tilde{F}^{\mu \nu}=0,
$$

where the second condition follows from the first due to self-duality. The vanishing of the field strength contracted with itself implies that the gauge field has zero action, as does the gravity solution. ${ }^{4}$ The field strength also has a singularity as $r \rightarrow a$, mirroring

\footnotetext{
${ }^{4}$ The reader might be concerned that $F^{2}=0$ implies that $F=0$, but the complexification of the field strength induced by the coordinate transformation of eq. (3.18) allows non-trivial field strengths to have vanishing action.
} 


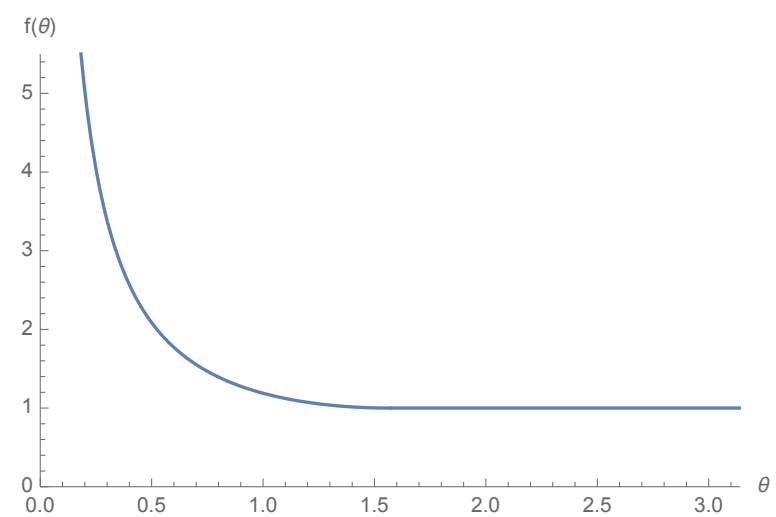

Figure 1. The function of eq. (3.27) entering the gauge transformation of eqs. (3.25), (3.26).

the bolt singularity in the gravity theory. However, unlike in the gravity case, where the bolt is a genuine singularity in the metric, its gauge theory counterpart is entirely spurious, having been introduced by the coordinate transformation of eq. (3.18). Without this transformation, the gauge field is well-behaved, and we stress again that it lives in flat space.

A noteworthy feature of the single copy field (in the polar coordinates) is the presence of an apparent singularity as $\theta \rightarrow 0$, which at first glance looks like the so-called Dirac string singularity of a magnetic monopole [92], associated with non-zero magnetic charge. However, we can completely remove this singularity using a (globally defined) gauge transformation

$$
A_{\mu} \rightarrow A_{\mu}^{\prime} \equiv A_{\mu}(r, \theta, \psi, \phi)-\partial_{\mu} \alpha(r, \theta, \psi, \phi)
$$

where

$$
\alpha=\frac{2 e^{i \psi}}{\sqrt{r^{4}-a^{4}}} f(\theta)
$$

and

$$
f(\theta)= \begin{cases}\csc \theta, & \theta \leq \frac{\pi}{2} \\ 1, & \theta>\frac{\pi}{2}\end{cases}
$$

A plot of this function is shown in figure 1. Both the function and its first derivative are continuous at $\theta=\pi / 2$. One then has

$$
A_{\mu}^{\prime}=\left\{\begin{array}{c}
\frac{e^{i \psi}}{\sqrt{r^{4}-a^{4}}}\left(\frac{2 r^{3} \tan (\theta / 2)}{r^{4}-a^{4}},-\frac{1}{2} \tan ^{2}(\theta / 2),-i \tan (\theta / 2),-\frac{i}{2} \sin \theta\right), \\
\theta \leq \pi / 2 ; \\
\frac{e^{i \psi}}{\sqrt{r^{4}-a^{4}}}\left(\frac{2 r^{3}(2-\cot (\theta / 2))}{r^{4}-a^{4}},-\frac{1}{2} \cot ^{2}(\theta / 2),-i(2-\cot (\theta / 2)),-\frac{i}{2} \sin \theta\right), \\
\theta>\pi / 2 .
\end{array}\right.
$$




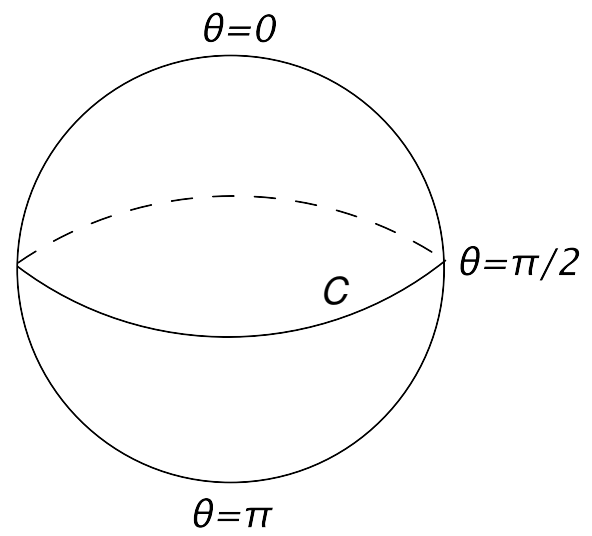

Figure 2. Northern and southern hemispheres for fixed $r$ and $\psi$, and the equator $C$.

It is easily checked that $A_{\mu}^{\prime}$ is continuous at $\theta=\pi / 2$, and non-singular for all $\theta \in[0, \pi]$. The fact that the string-like singularity has been removed suggests that there is no magnetic charge. To verify this explicity, consider a surface at fixed $\psi$ (which plays the role of the time coordinate), and $r$. This is a sphere defined by spherical polar coordinates $(\theta, \phi)$, as shown in figure 2. The magnetic charge is associated with the first Chern number

$$
c_{1}=\frac{1}{4 \pi} \int_{\Sigma} F_{\mu \nu} d \Sigma^{\mu \nu}
$$

where the closed 2-surface $\Sigma$ is the above sphere, and we may choose $\psi=0$ without loss of generality. We may carry out the surface integral by splitting it into two pieces, for the northern and southern hemispheres respectively. Using Stoke's theorem, we then have

$$
c_{1}=\int_{C} d x^{\mu} \delta A_{\mu}^{\prime}=0
$$

where $\delta A_{\mu}^{\prime}$ is the difference in the (transformed) gauge field in the northern and southern hemispheres, and the line integral is around the equator $C$. The second equality follows given that $\delta A_{\mu}^{\prime}=0$ on $C$.

We should not be surprised that the magnetic charge is zero. Taking $\psi$ as a (periodic) time coordinate, we see from eq. (3.21) that the electric field points tangential to the $S^{2}$ surface spanned by $(\theta, \phi)$. Thus, there is no electric flux through a closed surface enclosing the single copy solution, implying (via Gauss' Law) that the electric charge is zero. Selfduality then dictates that the magnetic charge must also be zero. This is also consistent with the fact that field-strength falls off in a dipole-like way $\sim r^{-3}$ (for fixed $\psi$ ) at large distances: a dipole has no net charge.

We have seen that the single copy gauge field has vanishing action, and the natural question arises of whether it can be given an instanton interpretation, analogous to that of its gravitational counterpart. However, there can be no abelian-like instanton solutions, owing to the fact that one requires a solution to be pure gauge at infinity, such that there is a topologically nontrivial map from the boundary of spacetime $\left(S^{3}\right)$ to the gauge group. There are no such maps ${ }^{5}$ to the abelian group U(1). Another way to see the absence of

\footnotetext{
${ }^{5}$ Put more formally, the third homotopy group $\pi_{3}[\mathrm{U}(1)]$ is trivial.
} 
instanton solutions is to note that they can be labelled by the second Chern number

$$
c_{2}=-\frac{1}{8 \pi^{2}} \int_{\mathbb{R}^{4}} \operatorname{Tr}[F \wedge F]=-\frac{1}{8 \pi^{2}} \int_{\partial \mathbb{R}^{4} \equiv S^{3}} \operatorname{Tr}[A \wedge F],
$$

where we use differential form notation, such that $F=d A$ is the field strength. The integral on the right-hand side vanishes, due to the fact that the integrand is zero for an abelian theory (as we have verified by explicit calculation).

The vanishing of the first and second Chern numbers mean that there is no nontrivial topological character to the single copy gauge field, which can be related directly to the gravity solution. We saw that the Eguchi-Hanson spacetime is topologically trivial before restricting the period of $\psi$ as in eq. (3.7). Here we have taken the single copy without imposing any restrictions on the background (flat) spacetime that the gauge theory lives in. Thus, the topological triviality of the single copy is entirely consistent with the similar behaviour of the (unrestricted) gravity solution. We may then ask whether it is possible to obtain topologically nontrivial behaviour by restricting the range of $\psi$ in the electromagnetic theory. However, the above arguments are unmodified by such a restriction: $\psi$ plays the role of a time coordinate, and the lack of magnetic charge at one time persists at later times. Furthermore, the obstruction to obtaining a nontrivial second Chern number is unchanged, in that the integrand in eq. (3.31) remains zero. Thus, there seems no way to obtain a gauge theory counterpart to the non-trivial topology of the gravity solution after removing the bolt singularity in the latter. We stress again that this is not surprising: non-trivial topology in the gravity theory arises upon removing the bolt singularity. The latter has no meaning in the gauge theory i.e. the apparent singularity at $r=a$ can be removed by a coordinate transformation, which is not the same as a gauge transformation. There is therefore no non-trivial topological character to the gauge field.

In order to corroborate the above discussion, it is instructive to plot the electric field (related to the magnetic field by self-duality) obtained from the single copy gauge field. To this end, we use Cartesian coordinates in Minkowski signature, obtained from the coordinates $(t, x, y, z)$ in eq. (2.12) by sending $t \rightarrow i t$. In figure 3 , we show the electric field in the plane $z=0$, for two different times. At time $t=0$, the field indeed appears purely dipole-like. As time increases, a disturbance propogates outwards at the speed of light, such that the field is singular on the circular contour $x^{2}+y^{2}=1$. Outside this region, however, the dipole-like form remains. The field in other planes is more complicated. As an example, we show the field in the $(x, z)$ plane in figure 4. Again there is a lightcone singularity at unit radius in the plane for $t=1$, but now the centre of the dipole-like behaviour is progressively shifted as time progresses. In all of the field configurations shown, there is clearly no net electric charge and thus, by self-duality, no magnetic charge.

\section{Conclusion}

In this paper, we have examined the double copy for exact classical solutions of gauge theory and gravity that are Kerr-Schild, but also self-dual. For this special class, one may consider a harmonic function in light-cone coordinates, and interpret the Kerr-Schild vector 


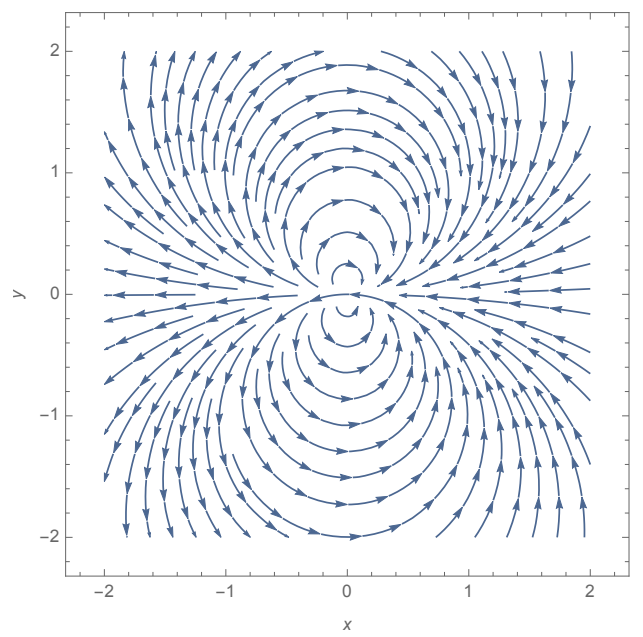

(a)

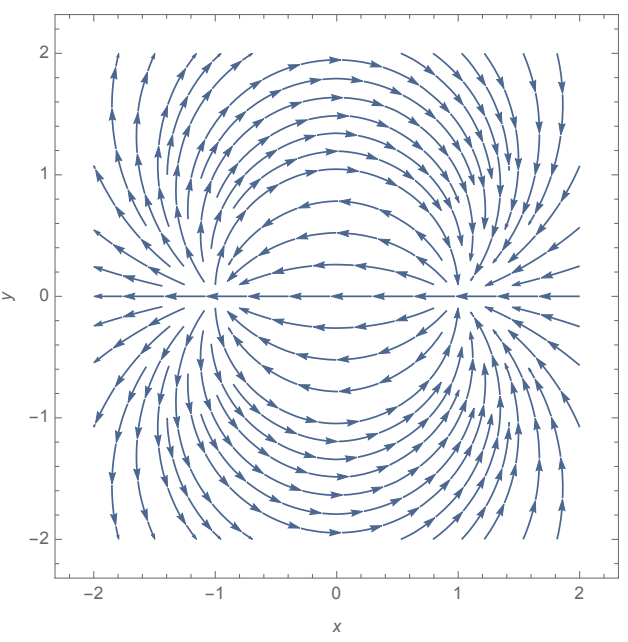

(b)

Figure 3. The real part of the electric field of the Eguchi-Hanson single copy, using Cartesian coordinates in Minkowski signature: (a) for $(t, z)=(0,0)$; (b) for $(t, z)=(1,0)$. The imaginary part can be obtained by rotating the figures by $90^{\circ}$.

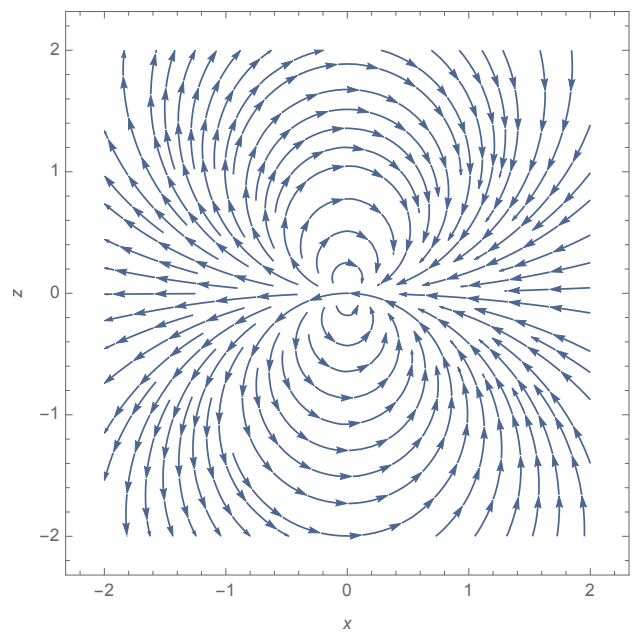

(a)

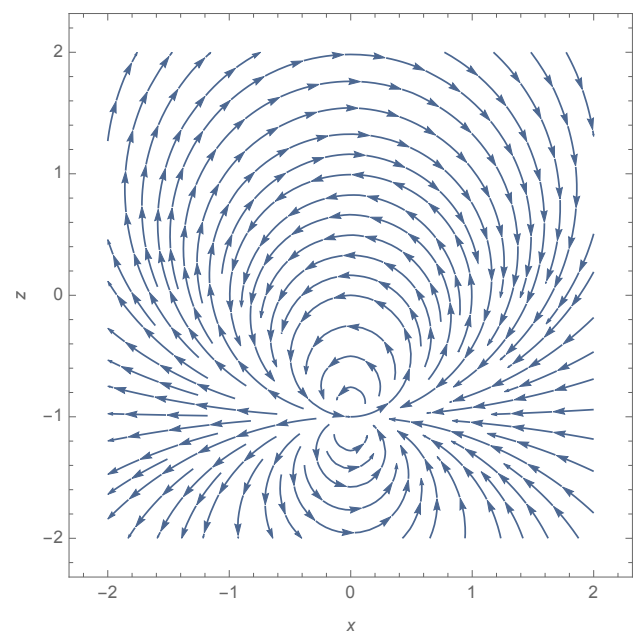

(b)

Figure 4. The real part of the electric field of the Eguchi-Hanson single copy, using Cartesian coordinates in Minkowski signature: (a) for $(t, y)=(0,0)$; (b) for $(t, y)=(1,0)$. The imaginary part is zero.

as a certain differential operator $\hat{k}_{\mu}$, which acts on the harmonic function to produce gauge and graviton fields. The harmonic function and gauge field are then the zeroth and single copy of the graviton respectively.

As an example of this approach, we have constructed a single copy of the well-known Eguchi-Hanson solution, which has been widely studied over the years as a gravitational instanton. The Eguchi-Hanson instanton has non-trivial topological structure, after removal of the so-called bolt singularity occuring in the metric. Before removal of this singularity 
however, the Euler characteristic and Hirzebruch signature of the spacetime are both zero, implying trivial topology. Consistent with this, we find that the single copy gauge field is also topologically trivial, as it must be given that it is abelian-like, and thus cannot exhibit nonzero instanton number if the boundary of spacetime is $S^{3}$. The magnetic charge is also zero, matching the fact that the field has dipole-like behaviour.

Our results are interesting in that one might have expected the single copy of the Eguchi-Hanson metric to be a non-abelian instanton, such as the well-known BPST instanton of ref. [81]. Indeed, it was the discovery of the latter solution that motivated the original study that led to the Eguchi-Hanson metric [78]. We find instead a single copy field that is abelian-like, and has trivial topology, and it is not the first time that the single copy of a well-known gravity solution has turned out to be simpler than expected. Before ref. [44], it was expected by many (including one of the present authors) that the single copy of the Schwarzschild solution would be a non-abelian object e.g. a monopole. However, the single copy turns out to be a simple Coulomb charge. This was later made sense of by studying the Taub-NUT solution [45], which confirmed that the single copy maps mass and NUT charge in the gravity theory to electric and magnetic charge, respectively, in the gauge theory. For Eguchi-Hanson, there is zero NUT charge and mass, and thus we indeed expect no electric or magnetic charge in its single copy.

Our results will also be useful in guiding studies of nonperturbative aspects of the double copy. Currently, all known examples of the copy involve objects cast in perturbation theory, or involving positive powers of the coupling constant. It is not known whether the double copy is a truly nonperturbative statement, expressing a much deeper relationship between gauge and gravity theories than previously thought. Furthermore, it is not even clear how to systematically formulate such a relationship. One way forward might be to catalogue known nonperturbative (or strong coupling) solutions between different theories, before matching them up. To this end, some strong coupling solutions of biadjoint scalar theory have been recently derived in refs. [93, 94].

Another way forward might be to use symmetries to characterise nonperturbative behaviour on both sides of the double copy correspondence, and to match up solutions according to e.g. topological information. Here, we have found that the creation of topological charge in the gravity theory does not necessarily have a unique counterpart in the gauge theory. The removal of the bolt singularity in the Eguchi-Hanson metric can be seen as a boundary condition, which has no immediate analogue in gauge theory.

There remains the possibility that there may yet be topologically nontrivial non-abelian solutions that map to Eguchi-Hanson, in addition to the abelian-like gauge field considered here. The fact that both an abelian and non-abelian quantity may map to the same gravitational quantity is possible given that colour structure is stripped off upon performing the double copy, and indeed such behaviour has been seen before in the study of infrared singularities [36]. One way to proceed may be to embed the abelian-like field seen here in a fully non-abelian setting using the well-known t'Hooft ansatz [95].

In summary, the study of classical aspects of the double copy, including possible nonperturbative aspects, is in its infancy, with many exciting avenues still to be explored. We hope that our present study sheds some light on some of these possibilities. 


\section{Acknowledgments}

We thank Joe Hayling for remarks that initiated this study, as well as Andreas Brandhuber, Gabriele Travaglini and Costis Papageorgakis for numerous illuminating chats. We are also grateful to Donal O'Connell and Ricardo Monteiro for discussions and collaboration on related topics. CDW and DB are supported by the U.K. Science and Technology Facilities Council (STFC). AL is supported in part by the Department of Energy under Award Number DESC000993.

Open Access. This article is distributed under the terms of the Creative Commons Attribution License (CC-BY 4.0), which permits any use, distribution and reproduction in any medium, provided the original author(s) and source are credited.

\section{References}

[1] Z. Bern, J.J.M. Carrasco and H. Johansson, New relations for gauge-theory amplitudes, Phys. Rev. D 78 (2008) 085011 [arXiv: 0805.3993] [INSPIRE].

[2] Z. Bern, J.J.M. Carrasco and H. Johansson, Perturbative quantum gravity as a double copy of gauge theory, Phys. Rev. Lett. 105 (2010) 061602 [arXiv:1004.0476] [INSPIRE].

[3] Z. Bern, T. Dennen, Y.-t. Huang and M. Kiermaier, Gravity as the square of gauge theory, Phys. Rev. D 82 (2010) 065003 [arXiv: 1004.0693] [InSPIRE].

[4] N.E.J. Bjerrum-Bohr, P.H. Damgaard and P. Vanhove, Minimal basis for gauge theory amplitudes, Phys. Rev. Lett. 103 (2009) 161602 [arXiv:0907.1425] [INSPIRE].

[5] S. Stieberger, Open \& closed vs. pure open string disk amplitudes, arXiv:0907.2211 [INSPIRE].

[6] N.E.J. Bjerrum-Bohr, P.H. Damgaard, T. Sondergaard and P. Vanhove, Monodromy and Jacobi-like relations for color-ordered amplitudes, JHEP 06 (2010) 003 [arXiv:1003.2403] [INSPIRE].

[7] B. Feng, R. Huang and Y. Jia, Gauge amplitude identities by on-shell recursion relation in S-matrix program, Phys. Lett. B 695 (2011) 350 [arXiv: 1004.3417] [inSPIRE].

[8] S.H. Henry Tye and Y. Zhang, Dual identities inside the gluon and the graviton scattering amplitudes, JHEP 06 (2010) 071 [Erratum ibid. 04 (2011) 114] [arXiv:1003.1732] [INSPIRE].

[9] C.R. Mafra, O. Schlotterer and S. Stieberger, Explicit BCJ numerators from pure spinors, JHEP 07 (2011) 092 [arXiv: 1104.5224] [INSPIRE].

[10] R. Monteiro and D. O'Connell, The kinematic algebra from the self-dual sector, JHEP 07 (2011) 007 [arXiv:1105.2565] [INSPIRE].

[11] N.E.J. Bjerrum-Bohr, P.H. Damgaard, R. Monteiro and D. O'Connell, Algebras for amplitudes, JHEP 06 (2012) 061 [arXiv:1203.0944] [INSPIRE].

[12] H. Kawai, D.C. Lewellen and S.H.H. Tye, A relation between tree amplitudes of closed and open strings, Nucl. Phys. B 269 (1986) 1 [INSPIRE].

[13] Z. Bern et al., On the relationship between Yang-Mills theory and gravity and its implication for ultraviolet divergences, Nucl. Phys. B 530 (1998) 401 [hep-th/9802162] [INSPIRE]. 
[14] M.B. Green, J.H. Schwarz and L. Brink, $N=4$ Yang-Mills and $N=8$ supergravity as limits of string theories, Nucl. Phys. B 198 (1982) 474 [INSPIRE].

[15] Z. Bern, J.S. Rozowsky and B. Yan, Two loop four gluon amplitudes in $N=4$ super-Yang-Mills, Phys. Lett. B 401 (1997) 273 [hep-ph/9702424] [INSPIRE].

[16] J.J. Carrasco and H. Johansson, Five-point amplitudes in $N=4$ Super-Yang-Mills theory and $N=8$ supergravity, Phys. Rev. D 85 (2012) 025006 [arXiv:1106.4711] [INSPIRE].

[17] J.J.M. Carrasco, M. Chiodaroli, M. Günaydin and R. Roiban, One-loop four-point amplitudes in pure and matter-coupled $N \leq 4$ supergravity, JHEP 03 (2013) 056 [arXiv: 1212.1146] [INSPIRE].

[18] C.R. Mafra and O. Schlotterer, The structure of n-point one-loop open superstring amplitudes, JHEP 08 (2014) 099 [arXiv: 1203.6215] [INSPIRE].

[19] R.H. Boels, R.S. Isermann, R. Monteiro and D. O'Connell, Colour-kinematics duality for one-loop rational amplitudes, JHEP 04 (2013) 107 [arXiv:1301.4165] [INSPIRE].

[20] N.E.J. Bjerrum-Bohr, T. Dennen, R. Monteiro and D. O'Connell, Integrand oxidation and one-loop colour-dual numerators in $N=4$ gauge theory, JHEP 07 (2013) 092 [arXiv: 1303.2913] [INSPIRE].

[21] Z. Bern, S. Davies, T. Dennen, Y.-T. Huang and J. Nohle, Color-kinematics duality for pure Yang-Mills and gravity at one and two loops, Phys. Rev. D 92 (2015) 045041 [arXiv: 1303.6605] [INSPIRE].

[22] Z. Bern, S. Davies and T. Dennen, The ultraviolet structure of half-maximal supergravity with matter multiplets at two and three loops, Phys. Rev. D 88 (2013) 065007 [arXiv:1305.4876] [INSPIRE].

[23] J. Nohle, Color-kinematics duality in one-loop four-gluon amplitudes with matter, Phys. Rev. D 90 (2014) 025020 [arXiv: 1309.7416] [INSPIRE].

[24] Z. Bern et al., Ultraviolet properties of $N=4$ supergravity at four loops, Phys. Rev. Lett. 111 (2013) 231302 [arXiv:1309.2498] [INSPIRE].

[25] S.G. Naculich, H. Nastase and H.J. Schnitzer, All-loop infrared-divergent behavior of most-subleading-color gauge-theory amplitudes, JHEP 04 (2013) 114 [arXiv:1301.2234] [INSPIRE].

[26] Y.-J. Du, B. Feng and C.-H. Fu, Dual-color decompositions at one-loop level in Yang-Mills theory, JHEP 06 (2014) 157 [arXiv: 1402.6805] [INSPIRE].

[27] C.R. Mafra and O. Schlotterer, Towards one-loop SYM amplitudes from the pure spinor BRST cohomology, Fortsch. Phys. 63 (2015) 105 [arXiv:1410.0668] [INSPIRE].

[28] Z. Bern, S. Davies and T. Dennen, Enhanced ultraviolet cancellations in $\mathcal{N}=5$ supergravity at four loops, Phys. Rev. D 90 (2014) 105011 [arXiv: 1409.3089] [INSPIRE].

[29] C.R. Mafra and O. Schlotterer, Two-loop five-point amplitudes of super Yang-Mills and supergravity in pure spinor superspace, JHEP 10 (2015) 124 [arXiv: 1505.02746] [INSPIRE].

[30] S. He, R. Monteiro and O. Schlotterer, String-inspired BCJ numerators for one-loop MHV amplitudes, JHEP 01 (2016) 171 [arXiv: 1507.06288] [INSPIRE].

[31] Z. Bern, S. Davies and J. Nohle, Double-copy constructions and unitarity cuts, Phys. Rev. D 93 (2016) 105015 [arXiv: 1510.03448] [INSPIRE]. 
[32] G. Mogull and D. O'Connell, Overcoming obstacles to colour-kinematics duality at two loops, JHEP 12 (2015) 135 [arXiv: 1511.06652] [INSPIRE].

[33] M. Chiodaroli, M. Günaydin, H. Johansson and R. Roiban, Spontaneously broken Yang-Mills-Einstein supergravities as double copies, JHEP 06 (2017) 064 [arXiv: 1511.01740] [INSPIRE].

[34] Z. Bern et al., Five-loop four-point integrand of $N=8$ supergravity as a generalized double copy, Phys. Rev. D 96 (2017) 126012 [arXiv:1708.06807] [inSPIRE].

[35] H. Johansson and A. Ochirov, Color-kinematics duality for QCD amplitudes, JHEP 01 (2016) 170 [arXiv: 1507.00332] [INSPIRE].

[36] S. Oxburgh and C.D. White, BCJ duality and the double copy in the soft limit, JHEP 02 (2013) 127 [arXiv:1210.1110] [INSPIRE].

[37] C.D. White, Factorization properties of soft graviton amplitudes, JHEP 05 (2011) 060 [arXiv:1103.2981] [INSPIRE].

[38] S. Melville, S.G. Naculich, H.J. Schnitzer and C.D. White, Wilson line approach to gravity in the high energy limit, Phys. Rev. D 89 (2014) 025009 [arXiv: 1306.6019] [INSPIRE].

[39] A. Luna, S. Melville, S.G. Naculich and C.D. White, Next-to-soft corrections to high energy scattering in QCD and gravity, JHEP 01 (2017) 052 [arXiv: 1611.02172] [INSPIRE].

[40] R. Saotome and R. Akhoury, Relationship Between Gravity and Gauge Scattering in the High Energy Limit, JHEP 01 (2013) 123 [arXiv:1210.8111] [INSPIRE].

[41] A. Sabio Vera, E. Serna Campillo and M.A. Vazquez-Mozo, Color-kinematics duality and the Regge limit of inelastic amplitudes, JHEP 04 (2013) 086 [arXiv:1212.5103] [INSPIRE].

[42] H. Johansson et al., Color-kinematics duality in multi-Regge kinematics and dimensional reduction, JHEP 10 (2013) 215 [arXiv:1307.3106] [INSPIRE].

[43] H. Johansson, A. Sabio Vera, E. Serna Campillo and M.A. Vazquez-Mozo, Color-kinematics duality and dimensional reduction for graviton emission in Regge limit, arXiv:1310.1680 [INSPIRE].

[44] R. Monteiro, D. O'Connell and C.D. White, Black holes and the double copy, JHEP 12 (2014) 056 [arXiv: 1410.0239] [INSPIRE].

[45] A. Luna, R. Monteiro, D. O'Connell and C.D. White, The classical double copy for Taub-NUT spacetime, Phys. Lett. B 750 (2015) 272 [arXiv:1507.01869] [INSPIRE].

[46] A. Luna et al., The double copy: Bremsstrahlung and accelerating black holes, JHEP 06 (2016) 023 [arXiv: 1603.05737] [INSPIRE].

[47] A.K. Ridgway and M.B. Wise, Static spherically symmetric Kerr-Schild metrics and implications for the classical double copy, Phys. Rev. D 94 (2016) 044023 [arXiv: 1512.02243] [INSPIRE].

[48] A. Anastasiou et al., Yang-Mills origin of gravitational symmetries, Phys. Rev. Lett. 113 (2014) 231606 [arXiv: 1408.4434] [INSPIRE].

[49] L. Borsten and M.J. Duff, Gravity as the square of Yang-Mills?, Phys. Scripta 90 (2015) 108012 [arXiv: 1602.08267 ] [INSPIRE].

[50] A. Anastasiou et al., Twin supergravities from Yang-Mills theory squared, Phys. Rev. D 96 (2017) 026013 [arXiv: 1610.07192] [INSPIRE]. 
[51] A. Anastasiou et al., Are all supergravity theories Yang-Mills squared?, Nucl. Phys. B 934 (2018) 606 [arXiv: 1707.03234] [INSPIRE].

[52] G.L. Cardoso, S. Nagy and S. Nampuri, A double copy for $\mathcal{N}=2$ supergravity: a linearised tale told on-shell, JHEP 10 (2016) 127 [arXiv:1609.05022] [INSPIRE].

[53] L. Borsten, $D=6, \mathcal{N}=(2,0)$ and $\mathcal{N}=(4,0)$ theories, Phys. Rev. D 97 (2018) 066014 [arXiv: 1708.02573] [INSPIRE].

[54] A. Anastasiou et al., The mile high magic pyramid, arXiv:1711.08476 [INSPIRE].

[55] A. Anastasiou et al., Gravity as gauge theory squared: a ghost story, Phys. Rev. Lett. 121 (2018) 211601 [arXiv:1807.02486] [INSPIRE].

[56] G. Lopes Cardoso, G. Inverso, S. Nagy and S. Nampuri, Comments on the double copy construction for gravitational theories, PoS (CORFU2017) 177 [arXiv: 1803.07670] [INSPIRE].

[57] W.D. Goldberger and A.K. Ridgway, Radiation and the classical double copy for color charges, Phys. Rev. D 95 (2017) 125010 [arXiv:1611.03493] [InSPIRE].

[58] W.D. Goldberger, S.G. Prabhu and J.O. Thompson, Classical gluon and graviton radiation from the bi-adjoint scalar double copy, Phys. Rev. D 96 (2017) 065009 [arXiv:1705.09263] [INSPIRE].

[59] W.D. Goldberger and A.K. Ridgway, Bound states and the classical double copy, Phys. Rev. D 97 (2018) 085019 [arXiv: 1711.09493] [INSPIRE].

[60] W.D. Goldberger, J. Li and S.G. Prabhu, Spinning particles, axion radiation and the classical double copy, Phys. Rev. D 97 (2018) 105018 [arXiv:1712.09250] [InSPIRE].

[61] A. Luna et al., Perturbative spacetimes from Yang-Mills theory, JHEP 04 (2017) 069 [arXiv: 1611.07508] [INSPIRE].

[62] A. Luna, I. Nicholson, D. O'Connell and C.D. White, Inelastic black hole scattering from charged scalar amplitudes, JHEP 03 (2018) 044 [arXiv:1711.03901] [INSPIRE].

[63] C.-H. Shen, Gravitational radiation from color-kinematics duality, JHEP 11 (2018) 162 [arXiv: 1806. 07388] [INSPIRE].

[64] M. Levi, Effective field theories of post-newtonian gravity: a comprehensive review, arXiv: 1807.01699 [INSPIRE].

[65] J. Plefka, J. Steinhoff and W. Wormsbecher, Effective action of dilaton gravity as the classical double copy of Yang-Mills theory, arXiv:1807.09859 [INSPIRE].

[66] C. Cheung, I.Z. Rothstein and M.P. Solon, From scattering amplitudes to classical potentials in the post-Minkowskian expansion, Phys. Rev. Lett. 121 (2018) 251101 [arXiv:1808.02489] [INSPIRE].

[67] M. Carrillo González, R. Penco and M. Trodden, Radiation of scalar modes and the classical double copy, JHEP 11 (2018) 065 [arXiv: 1809.04611] [INSPIRE].

[68] F. Cachazo, S. He and E.Y. Yuan, Scattering of massless particles in arbitrary dimensions, Phys. Rev. Lett. 113 (2014) 171601 [arXiv:1307.2199] [INSPIRE].

[69] F. Cachazo, S. He and E.Y. Yuan, Scattering of massless particles: scalars, gluons and gravitons, JHEP 07 (2014) 033 [arXiv: 1309.0885] [inSPIRE].

[70] L. Mason and D. Skinner, Ambitwistor strings and the scattering equations, JHEP 07 (2014) 048 [arXiv: 1311.2564] [INSPIRE]. 
[71] T. Adamo, E. Casali, L. Mason and S. Nekovar, Scattering on plane waves and the double copy, Class. Quant. Grav. 35 (2018) 015004 [arXiv: 1706.08925] [INSPIRE].

[72] N. Bahjat-Abbas, A. Luna and C.D. White, The Kerr-Schild double copy in curved spacetime, JHEP 12 (2017) 004 [arXiv:1710.01953] [INSPIRE].

[73] M. Carrillo-González, R. Penco and M. Trodden, The classical double copy in maximally symmetric spacetimes, JHEP 04 (2018) 028 [arXiv:1711.01296] [INSPIRE].

[74] K. Lee, Kerr-Schild double field theory and classical double copy, JHEP 10 (2018) 027 [arXiv: 1807.08443] [INSPIRE].

[75] R. Monteiro, I. Nicholson and D. O'Connell, Spinor-helicity and the algebraic classification of higher-dimensional spacetimes, arXiv:1809.03906 [INSPIRE].

[76] R. Monteiro and D. O'Connell, The kinematic algebras from the scattering equations, JHEP 03 (2014) 110 [arXiv:1311.1151] [INSPIRE].

[77] K.P. Tod, Self-dual Kerr-Schild metrics and null Maxwell fields, J. Math. Phys. 23 (1982) 1147.

[78] T. Eguchi and A.J. Hanson, Asymptotically flat selfdual solutions to euclidean gravity, Phys. Lett. B 74 (1978) 249.

[79] T. Eguchi and A.J. Hanson, Selfdual solutions to Euclidean gravity, Annals Phys. 120 (1979) 82 [INSPIRE].

[80] T. Eguchi and A.J. Hanson, Gravitational instantons, Gen. Rel. Grav. 11 (1979) 315 [INSPIRE].

[81] A.A. Belavin et al., Pseudoparticle solutions of the Yang-Mills equations, Phys. Lett. B 59 (1975) 85 [INSPIRE].

[82] T. Ortin, Gravity and strings, Cambridge Monographs on Mathematical Physics, Cambridge University Press, Cambridge U.K. (2015).

[83] S. Lee, R. Roychowdhury and H.S. Yang, Test of emergent gravity, Phys. Rev. D 88 (2013) 086007 [arXiv: 1211.0207] [INSPIRE].

[84] J. Lee and H.S. Yang, Quantized Kähler geometry and quantum gravity, J. Korean Phys. Soc. 72 (2018) 1421 [arXiv: 1804.09171] [INSPIRE].

[85] A.H. Taub, Empty space-times admitting a three parameter group of motions, Ann. Math. $\mathbf{5 3}$ (1951) 472 .

[86] E. Newman, L. Tamburino and T. Unti, Empty-space generalization of the Schwarzschild metric, J. Math. Phys. 4 (1963) 915.

[87] A. Parkes, A cubic action for selfdual Yang-Mills, Phys. Lett. B 286 (1992) 265 [hep-th/9203074] [INSPIRE].

[88] G. 't Hooft, A physical interpretation of gravitational instantons, Nucl. Phys. B 315 (1989) 517 [INSPIRE].

[89] T. Eguchi, P.B. Gilkey and A.J. Hanson, Gravitation, gauge theories and differential geometry, Phys. Rept. 66 (1980) 213 [InSPIRE].

[90] G.W. Gibbons and S.W. Hawking, Classification of gravitational instanton symmetries, Commun. Math. Phys. 66 (1979) 291 [InSPIRE].

[91] G. Burnett-Stuart, Sparling-Tod metric = Eguchi-Hanson, Twistor Newsletter 9 (1979) 6. 
[92] P.A.M. Dirac, Quantised singularities in the electromagnetic field,, Proc. Roy. Soc. Lond. A 133 (1931) 60 [INSPIRE].

[93] C.D. White, Exact solutions for the biadjoint scalar field, Phys. Lett. B 763 (2016) 365 [arXiv: 1606. 04724] [INSPIRE].

[94] P.-J. De Smet and C.D. White, Extended solutions for the biadjoint scalar field, Phys. Lett. B 775 (2017) 163 [arXiv: 1708.01103] [INSPIRE].

[95] G. 't Hooft, Computation of the quantum effects due to a four-dimensional pseudoparticle, Phys. Rev. D 14 (1976) 3432 [Erratum ibid. D 18 (1978) 2199] [INSPIRE]. 